\section{MS33-O3 Structural differences in enantiopure and racemate organometallic complexes. Application to $\left[\left(\eta^{5}-\mathrm{C}_{5} \mathrm{Me}_{5}\right) \mathrm{RhCl}(\mathrm{PN})\right]^{+\mathrm{n}}$ complexes}

Fernando J. Lahoz ${ }^{1}$, Pilar García-Orduña ${ }^{1}$, María Carmona ${ }^{1}$, Ricardo Rodríguez ${ }^{1}$, Daniel Carmona ${ }^{1}$

1. Institute for Chemical Synthesis and Homogeneous Catalysis (ISQCH), CSIC - University of Zaragoza, C/ Pedro Cerbuna, 12 , 50009 Zaragoza (Spain)

email: lahoz@unizar.es

The use of metal-based catalysts is one of the most powerful strategies for synthesizing enantioenriched compounds by asymmetric catalysis. Our lab is focused in the development and structural characterization of different types of coordination complexes, based on $2^{\text {nd }}$ and $3^{\text {rd }}$ row late transition metals, and in their potential application as homogeneous catalyst precursors, trying to establish a clear correspondence between specific structural parameters and chemical and catalytic activity. This approach usually relies on organometallic complexes where the metal atoms are stereogenic centers. The asymmetric environment around the metal, i.e., the catalyst pocket in which catalysis occurs, may control the achieved enantioselectivity [1-3]. Therefore, the synthesis, isolation and the detailed structural characterization of enantiopure catalysts are primary goals in this research line.

Among the methodologies for enantiomer resolution, crystallization processes have been widely investigated, since Pasteur's famous separation of the sodium ammonium tartrate enantiomers [4]. Here we report on the characterization of a family of $\left[\left(\eta^{5}-\mathrm{C}_{5} \mathrm{Me}_{5}\right) \mathrm{RhCl}(\mathrm{PN})\right]^{+\mathrm{n}}$ complexes in different crystals. The metal is always a stereogenic center, and depending on the chiral nature of the employed ligand, the new compounds are mixtures of diastereomers and epimers at the metal. We have been able to obtain and separate red single crystals of enantiopure $R$ and $S$ at the metal derivatives, together with orange racemate crystals containing both $R$ and $S$ enantiomers.

A systematic analysis of their crystal and molecular structures has been performed; the pertinent structural parameters concerning metal bonding, ring puckering of chelate ligands' metallacycles, or intramolecular $\mathrm{N}-\mathrm{H} \cdots \mathrm{Cl}$ interactions have been analysed. The presence of solvent molecules in some crystals and their influence in intermolecular interactions will be discussed through Hirsfeld surface and two-dimensional fingerprint plot analysis.

[1] D. Carmona, P. Lamata, A. Sánchez, P. Pardo, R. Rodríguez, P. Ramírez, F.J. Lahoz, P. García-Orduña, L.A. Oro, Dalton Trans. 2014, 43, 15546. [2] D. Carmona, I. Méndez, R. Rodríguez, F.J. Lahoz, P. García-Orduña, L.A. Oro, Organometallics 2014, 33, 443. [3] A. Becerra, R. Contreras, D. Carmona, F.J. Lahoz, P. García-Orduña, Dalton Trans. 2013, 42, 11640. [4] L. Pasteur; Annales de Chimie et de Physique, 1848, 3,442 .

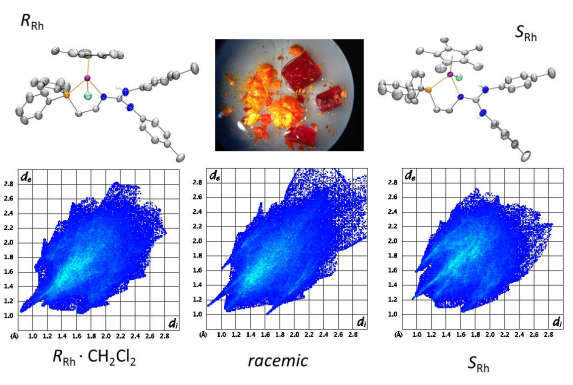

Figure 1. Molecular structure of $\mathrm{R}$ and $\mathrm{S}$ at-metal epimers in enantiopure crystals. Red (enantiopure) and orange (racemic) crystals. Fingerprint plots of rhodium complexes in the three crystals.

Keywords: Enantiopure, Racemic, Metal complexes, 